\title{
Spin structure of the pion in a light-cone representation
}

\author{
Bo-Qiang Maf \\ Institute of High Energy Physics, Academia Sinica, P.O.Box 918(4), \\ Beijing 100039, China
}

\begin{abstract}
The spin structure of the pion is discussed by transforming the wave function for the pion in the naive quark model into a lightcone representation. It is shown that there are higher helicity $\left(\lambda_{1}+\lambda_{2}= \pm 1\right)$ states in the full light-cone wave function for the pion besides the ordinary helicity $\left(\lambda_{1}+\lambda_{2}=0\right)$ component wave functions as a consequence from the Melosh rotation relating spin states in light-front dynamics and those in instantform dynamics. Some low energy properties of the pion, such as the electromagnetic form factor, the charged mean square radius, and the weak decay constant, could be interrelated in this representation with reasonable parameters.
\end{abstract}

To be published in Z.Phys.A

* Fellow of Alexander von Humboldt Foundation, at present in Institut für Theoretische Physik der Universität Frankfurt am Main, Postfach 1119 32, D-6000 Frankfurt, Germany 


\section{Introduction}

The light-cone formalism [1,2] provides a convenient framework for the relativistic description of hadrons in terms of quark and gluon degrees of freedom. There have been many studies on the valence state wave function for the pion in light-cone formalism [2-9] or in light-front dynamics[10-12]. It will be shown in this paper that the spin structure of the pion in light-cone formalism is quite different from that in the $\mathrm{SU}(6)$ naive quark model in considering the effect from the Melosh rotation [13,14] relating spin states in light-front dynamics and those in instant-form dynamics. A natural consequence is the presence of the higher helicity $\left(\lambda_{1}+\lambda_{2}= \pm 1\right)$ components in the full light-cone wave function for the pion besides the ordinary helicity $\left(\lambda_{1}+\lambda_{2}=0\right)$ components. In fact, the Melosh rotation has been applied to explain the "'proton spin puzzle" [14] and the emergency of the $\lambda_{1}+\lambda_{2}= \pm 1$ components in the pion has been also realized [7]. The purpose of this paper is to explore the explicit form of the light-cone wave function for the pion and the consequences of taking into account the $\lambda_{1}+\lambda_{2}= \pm 1$ components in the description of several low energy properties of the pion. It will be shown that the electromagnetic form factor, the charged mean square radius, and the weak decay constant could be reproduced by the harmonic oscillator wave function in the light-cone representation with very reasonable parameters by taking into account the contributions from the higher helicity states.

2. Intuitive argument and present status

We first give an intuitive picture to explain why there are higher helicity states in the full light-cone wave function for the pion. When a composite system is transformed from one reference frame to another frame, every constituent's spin will undergo a Wigner rotation [15], and these spin rotations may be not necessarily the same since the constituents may have different internal motions. In consequence the sum of the constituent's spin is not Lorentz invariant. Hence the pion, composed of two constituents with opposite spin in the rest frame, may have $\lambda_{1}+\lambda_{2}= \pm 1$ spin states in the infinite momentum frame, where $\lambda_{1}$ and $\lambda_{2}$ are the constituent's spins along the in- 
finite momentum frame; i.e., they are the helicities of the two constituents. We know that the instant-form dynamics in the infinite momentum frame is equivalent to light-front dynamics in an ordinary frame[16], thereby the spin structure for a composite system in light-front dynamics is quite different from that in the ordinary instant-form dynamics in considering the effect from the Wigner rotation.

It has been argued $[17,14]$ that the Melosh rotation [13] relating constituent quark and current quark can be understood as a special Wigner rotation relating spin states in instant-form dynamics and those in lightfront dynamics. The consequences from considering the Melosh rotation in the description of the pion low energy properties have been investigated in several papers $[8,9,12]$. In ref.[8] some kinematics corrections to the ordinary helicity $\left(\lambda_{1}+\lambda_{2}=0\right)$ component wave function were considered but the higher helicity $\left(\lambda_{1}+\lambda_{2}= \pm 1\right)$ states were unfortunately ignored. The pion wave function was represented in ref.[12] in terms of Pauli matrices and the physical implication is unclear. An explicit representation of the pion wave function was given by Kisslinger and Jacob [9] in terms of lightcone Dirac spinors in conjunction with a momentum space wave function evaluated from a light-cone Bethe-Salpeter formalism. However, the spin structure for the pion in the light-cone formalism, such as the presence of the higher helicity states, still remains unexplained.

3. The light-cone wave function for the pion

a) The spin wave function

We present in this paper an alternative light-cone representation of the pion full wave function by transforming the ordinary instant-form $\mathrm{SU}(6)$ quark model wave function for the pion into light-front dynamics. We start from the rest frame $\left(\vec{q}_{1}+\vec{q}_{2}=0\right)$ instant-form (T) spin wave function of the pion,

$$
\chi_{T}^{\pi}=\left(\chi_{1}^{\uparrow} \chi_{2}^{\downarrow}-\chi_{2}^{\uparrow} \chi_{1}^{\downarrow}\right) / \sqrt{2},
$$

In which $\chi_{i}^{\uparrow, \downarrow}$ is the two-component Pauli spinor and the two quarks have 4-momentum $q_{1}^{\mu}=\left(q^{0}, \vec{q}\right)$ and $q_{2}^{\mu}=\left(q^{0},-\vec{q}\right)$, with $q^{0}=\left(m^{2}+\vec{q}^{2}\right)^{1 / 2}$, respectively. The instant-form spin states $\mid J, s>_{T}$ and the front form (F) spin 
states $\mid J, \lambda>_{F}$ are related by a Wigner rotation $U^{J}$,

$$
\left|J, \lambda>_{F}=\sum_{s} U_{s \lambda}^{J}\right| J, s>_{T},
$$

and this rotation is called as Melosh rotation for spin- $1 / 2$ particles. One should transform both sides of eq.(1) simultaneously to get the light-cone spin wave function for the pion. For the left side, i.e., the pion, the transformation is particularly simple since the Wigner rotations are reduced to unity. For the right side, i.e., two spin-1/2 quarks, each particle instant-form and front-form spin states are related by the Melosh transformation[14],

$$
\begin{aligned}
& \chi^{\uparrow}(T)=w\left[\left(q^{+}+m\right) \chi^{\uparrow}(F)-q^{R} \chi^{\downarrow}(F)\right] ; \\
& \chi^{\downarrow}(T)=w\left[\left(q^{+}+m\right) \chi^{\downarrow}(F)+q^{L} \chi^{\uparrow}(F)\right],
\end{aligned}
$$

where $w=\left[2 q^{+}\left(q^{0}+m\right)\right]^{-1 / 2}, q^{R, L}=q^{1} \pm i q^{2}$, and $q^{+}=q^{0}+q^{3}$. Then we get the light-cone (or front form ) spin wave function for the pion,

$$
\chi^{\pi}\left(x, \vec{k}_{\perp}\right)=\sum_{\lambda_{1}, \lambda_{1}} C_{0}^{F}\left(x, \vec{k}_{\perp}, \lambda_{1}, \lambda_{2}\right) \chi_{1}^{\lambda_{1}}(F) \chi_{2}^{\lambda_{2}}(F)
$$

where the component coefficients $C_{J=0}^{F}\left(x, \vec{k}_{\perp}, \lambda_{1}, \lambda_{2}\right)$, when expressed in terms of the instant-form momentum $q^{\mu}=\left(q^{0}, \vec{q}\right)$, have the forms,

$$
\begin{gathered}
C_{0}^{F}\left(x, \vec{k}_{\perp}, \uparrow, \downarrow\right)=w^{1} w^{2}\left[\left(q_{1}^{+}+m\right)\left(q_{2}^{+}+m\right)-\vec{q}_{\perp}^{2}\right] / \sqrt{2} ; \\
C_{0}^{F}\left(x, \vec{k}_{\perp}, \downarrow, \uparrow\right)=-w^{1} w^{2}\left[\left(q_{1}^{+}+m\right)\left(q_{2}^{+}+m\right)-\vec{q}_{\perp}^{2}\right] / \sqrt{2} ; \\
C_{0}^{F}\left(x, \vec{k}_{\perp}, \uparrow, \uparrow\right)=w^{1} w^{2}\left[\left(q_{1}^{+}+m\right) q_{2}^{L}-\left(q_{2}^{+}+m\right) q_{1}^{L}\right] / \sqrt{2} ; \\
C_{0}^{F}\left(x, \vec{k}_{\perp}, \downarrow, \downarrow\right)=w^{1} w^{2}\left[\left(q_{1}^{+}+m\right) q_{2}^{R}-\left(q_{2}^{+}+m\right) q_{1}^{R}\right] / \sqrt{2} ;
\end{gathered}
$$

which satisfy the relation,

$$
\sum_{\lambda_{1}, \lambda_{2}} C_{0}^{F}\left(x, \vec{k}_{\perp}, \lambda_{1}, \lambda_{2}\right)^{*} C_{0}^{F}\left(x, \vec{k}_{\perp}, \lambda_{1}, \lambda_{2}\right)=1
$$

One sees that there are also two higher helicity $\left(\lambda_{1}+\lambda_{2}= \pm 1\right)$ components in the expression of the light-cone spin wave function of the pion besides the ordinary helicity $\left(\lambda_{1}+\lambda_{2}= \pm 1\right)$ components.

b) The momentum space wave function 
We still need to know the momentum space wave function. Unfortunately, there is no exact solution of the Bethe-Salpeter equation for the pion at present and in practice one often makes approximation to evaluate the momentum space wave function. A commonly used one for mesons is the harmonic oscillator wave function

$$
\varphi\left(\vec{q}^{2}\right)=A \exp \left(-\vec{q}^{2} / 2 \beta^{2}\right)
$$

which is a non-relativistic solution of the Bethe-Salpeter equation in an instantaneous approximation in the rest frame for mesons[18]. We indicate that the relation between the instant-form momentum $\vec{q}=\left(q^{3}, \vec{q}_{\perp}\right)$ and the light-cone momentum $\underline{k}=\left(x, \vec{k}_{\perp}\right)$ is by no means unique, and in practice one needs to construct models relating them. In this paper we adopt the connection[10-12]:

$$
\begin{gathered}
x=\left(q^{0}+q^{3}\right) / M ; \\
\vec{k}_{\perp}=\vec{q}_{\perp},
\end{gathered}
$$

in which $M$ satisfies

$$
M^{2}=\frac{\vec{k}_{\perp}^{2}+m^{2}}{x(1-x)}
$$

From eq.(8) we find,

$$
\begin{aligned}
& q^{0}=\left[x M+\left(m^{2}+\vec{k}_{\perp}^{2}\right) / x M\right] / 2 ; \\
& q^{3}=\left[x M-\left(m^{2}+\vec{k}_{\perp}^{2}\right) / x M\right] / 2,
\end{aligned}
$$

thus we obtain

$$
\begin{gathered}
q^{+}=x M \\
2 q^{+}\left(q^{0}+m\right)=(x M+m)^{2}+\vec{k}_{\perp}^{2} .
\end{gathered}
$$

We notice

$$
\vec{q}^{2}=\frac{\vec{k}_{\perp}^{2}+m^{2}}{4 x(1-x)}-m^{2}
$$

As there are ambiguities in extending the non-relativistic form wave function into a relativistic one, we find that there are three possible prescriptions for the transformed light-cone momentum space wave function in the literature: 
1. The Brodsky-Huang-Lepage (BHL) prescription [2]

$$
\varphi_{B H L}\left(x, \vec{k}_{\perp}\right)=A \exp \left[-\frac{m^{2}+\vec{k}_{\perp}^{2}}{8 \beta^{2} x(1-x)}\right]
$$

2. The Teren'ev-Karmanov (TK) prescription [10-11]

$$
\varphi_{T K}\left(x, \vec{k}_{\perp}\right)=A \sqrt{\frac{1}{2 x(1-x)}} \exp \left[-\frac{m^{2}+\vec{k}_{\perp}^{2}}{8 \beta^{2} x(1-x)}\right],
$$

where the factor $\sqrt{1 / 2 x(1-x)}$ arises from the jacobian relating $d^{3} \vec{q} / q^{0}$ and $d^{2} \vec{k}_{\perp} d x$;

3. The Chung-Coester-Polyzou (CCP) prescription [12]

$$
\varphi_{C C P}\left(x, \vec{k}_{\perp}\right)=A \sqrt{\frac{M}{4 x(1-x)}} \exp \left[-\frac{m^{2}+\vec{k}_{\perp}^{2}}{8 \beta^{2} x(1-x)}\right],
$$

where the factor $\sqrt{M / 4 x(1-x)}$ arises from the jacobian relating $d^{3} \vec{q}$ and $d^{2} \vec{k}_{\perp} d x$.

The three prescriptions differ in the factor related with the jacobian adopted:

$J_{B H L}^{2}=1, J_{T K}^{2}=1 / 2 x(1-x)$, and $J_{C C P}^{2}=M / 4 x(1-x)$.

c) Parameter fixing

Thereby we obtain the light-cone wave function for the pion

$$
\psi=\varphi \chi
$$

in which the parameters are the quark mass $m$, the harmonic scale $\beta$ and the normalization constant $\mathrm{A}$. The wave functions for the pion in previous work $[8-9,12]$ were shown to be successful in reproducing some low energy properties of the pion, such as the electromagnetic form factor, the charged mean square radius and the weak decay constant. We exam also these items for the wave functions in this paper. As the wave function, eq.[16], can be considered as a light-cone version of the $\mathrm{SU}(6)$ quark model wave function such as in ref.[18], we expect that the parameters $m$ and $\beta$ be not so much different from the values $m=330 \mathrm{MeV}$ and $\beta=220 \mathrm{MeV}$ in ref.[18,3]. The parameters are adjusted to fit the constraints adopted by Kisslinger and $\operatorname{Jacob}[9]$ : 
1. the normalization condition

$$
\int\left[d^{2} \vec{k}_{\perp} d x / 16 \pi^{3}\right] \psi^{*} \psi=\int\left[d^{2} \vec{k}_{\perp} d x / 16 \pi^{3}\right] \varphi^{*} \varphi=1,
$$

which is essentially a valence quark dominance assumption[8];

2. the weak decay constant $f_{\pi}=93 \mathrm{MeV}$ defined [2] from $\pi \rightarrow \mu \nu$ decay process by $<0\left|\bar{u} \gamma^{+}\left(1-\gamma_{5}\right) d\right| \pi>=-\sqrt{2} f_{\pi} p^{+}$, thus one obtains

$$
\int_{0}^{1} d x \int \frac{d^{2} \vec{k}_{\perp}}{16 \pi^{3}} \frac{\left(k_{1}^{+}+m\right)\left(k_{2}^{+}+m\right)-\vec{k}_{\perp}^{2}}{\left\{\left[\left(k_{1}^{+}+m\right)^{2}+\vec{k}_{\perp}^{2}\right]\left[\left(k_{2}^{+}+m\right)^{2}+\vec{k}_{\perp}^{2}\right]\right\}^{1 / 2}} \varphi=\frac{f_{\pi}}{2 \sqrt{3}} ;
$$

3. the charged mean square radius $\left\langle r_{\pi}^{2}>=0.439 \mathrm{fm}^{2}[19]\right.$ evaluated numerically from $<r_{\pi}^{2}>=-6 \partial F_{\pi}\left(Q^{2}\right) / \partial Q^{2}$ at $Q^{2}=0$.

We thus obtain $m=330 \mathrm{MeV}, \beta=290 \mathrm{MeV}$ for the BHL prescription; $m=330 \mathrm{MeV}, \beta=280 \mathrm{MeV}$ for the TK prescription; and $m=330 \mathrm{MeV}$, $\beta=270 \mathrm{MeV}$ for the $\mathrm{CCP}$ prescription. One sees, in comparison with the results in ref.[8,12], that the values of the parameters above are more close to those used in the conventional $\mathrm{SU}(6)$ quark model harmonic oscillator wave function[18,3].

4. The pion form factor

One advantage of light-front dynamics is that the Wigner rotation relating spin states in different frames is unity under kinematic Lorentz transformation, thereby the spin structure of hadrons are the same in different frames related by kinematic Lorentz transformation. The electromagnetic form factor can be calculated from the Drell-Yan-West formula[20]

$$
F\left(Q^{2}\right)=\sum_{\lambda_{i}} \int_{0}^{1} d x \int \frac{d^{2} \vec{k}_{\perp}}{16 \pi^{3}} \psi^{*}\left(x_{i}, \vec{k}_{\perp i}, \lambda_{i}\right) \psi\left(x_{i}, \overrightarrow{k^{\prime}} \perp i, \lambda_{i}\right),
$$

where $\vec{k}_{\perp i}=\vec{k}_{\perp i}-x_{i} \vec{q}_{\perp}+\vec{q}_{\perp}$ for the struck quark, $\vec{k}_{\perp i}=\vec{k}_{\perp i}-x_{i} \vec{q}_{\perp}$ for the spectator quarks, and the virtual photon momentum $q_{\mu}$ is specified with $q^{+}=0$ to eliminate the Z-graph contributions[1,2,21]. Other choice of $q_{\mu}$ will cause contributions from Z-graphs, and it should give the same result 
as that in the $q^{+}=0$ case if all the graphs are taken into account[22]. We thus obtain

$$
F\left(Q^{2}\right)=\int_{0}^{1} d x \int\left[d^{2} \vec{k}_{\perp} / 16 \pi^{3}\right] \mathcal{M} \varphi^{*}\left(\mathrm{x}, \overrightarrow{\mathrm{k}}_{\perp}\right) \varphi\left(\mathrm{x}, \overrightarrow{\mathrm{k}}_{\perp}\right)
$$

where $\vec{k}_{\perp}=\vec{k}_{\perp}+(1-x) \vec{q}_{\perp}$ is the internal quark transverse momentum of the struck pion in the center of mass frame, and

$$
\mathcal{M}=\frac{\left(\mathrm{a}_{1} \mathrm{a}_{2}-\overrightarrow{\mathrm{k}}_{\perp}^{2}\right)\left(\mathrm{a}_{1}^{\prime} \mathrm{a}_{2}^{\prime}-{\overrightarrow{\mathrm{k}^{\prime}}}_{\perp}^{2}\right)+\left(\mathrm{a}_{1}+\mathrm{a}_{2}\right)\left(\mathrm{a}_{1}^{\prime}+\mathrm{a}_{2}^{\prime}\right) \overrightarrow{\mathrm{k}}_{\perp} \cdot \overrightarrow{\mathrm{k}^{\prime}}}{\left[\left(\mathrm{a}_{1}^{2}+\overrightarrow{\mathrm{k}}_{\perp}^{2}\right)\left(\mathrm{a}_{2}^{2}+\overrightarrow{\mathrm{k}}_{\perp}^{2}\right)\left(\mathrm{a}_{1}^{\prime 2}+{\overrightarrow{\mathrm{k}^{\prime}}}_{\perp}^{2}\right)\left(\mathrm{a}_{2}^{\prime 2}+{\overrightarrow{\mathrm{k}^{\prime}}}_{\perp}^{2}\right)\right]^{1 / 2}}
$$

in which $a_{i}=k_{i}^{+}+m$ and $a_{i}^{\prime}=k_{i}^{+}+m$, is the contribution from the Melosh rotation. The above calculation does not (or less so severely) suffer from the flaws recognized in ref.[7] in evaluating the "'soft" form factor. One can easily find that our treatment of the Melosh rotation is also different from that of CCP by comparing eq.(21) above with eq.(19) in ref.[12]. This explains why our parameters differ to theirs. The comparisons of the calculated form factor with the data at low $Q^{2}$ are shown in fig.1. One sees, in combination with the three constraints, that several low energy properties of the pion, such as the electromagnetic form factor, the charged mean square radius, and the weak decay constant, can be interrelated in the three prescriptions with very reasonable parameters by taking into account the contributions from the higher helicity states.

Fig.2 presents the calculated form factor at higher $Q^{2}$ with the above wave functions in the constituent quark $q \bar{q}$ configuration. The calculated form factors, i.e., the unlabeled curves, fall off with $Q^{2}$ quickly by taking into account the contributions from higher helicity states. If we ignore the higher helicity states as was done in ref.[8], the calculated pion form factors, which should be approximately twice the magnitude of the curves labeled $\lambda_{1}+\lambda_{2}=0$, could be comparable in size to the data at currently available $Q^{2}$ in the three prescriptions. Thus the conclusion in ref.[8] might be altered if a different momentum space wave function other than the one specified there was used. By properly taking into account the contributions from the higher helicity states, we seem to have arrived the same conclusion as that 
in ref. [4,8] that the "'soft contributions" to the form factor are insufficient to explain the data at currently available large $Q^{2}$ and other QCD terms are necessary at high momentum transfer $\left(\geq\right.$ a few $\left.(\mathrm{GeV} / \mathrm{c})^{2}\right)$, even if we adopt different prescriptions for the pion light-cone wave function. Of course, the above conclusion is dependent on the specific form of the momentum space wave function and relies on the valence quark dominance assumption, thus may be altered if a different momentum space wave function, instead of eq.(7), is used or the valence dominance assumption, i.e., eq.(17), is removed. Nevertheless, we believe the results in fig. 2 imply that the constituent quark model in valence quark configuration could be valid at low energy scale even up to $Q^{2} \approx 1(\mathrm{GeV} / \mathrm{c})^{2}$ and that at higher resolution (i.e., $Q^{2} \geq$ a few $\left.(\mathrm{GeV} / \mathrm{c})^{2}\right)$ the contribution from considering the internal substructure of the constituent quarks should be further introduced in such picture like the valon model proposed by Hwa[25].

5. Remarks and summary

We indicate that our treatment of the Melosh rotation, though simply, is different from those in previous investigations. The introduction of the higher helicity states into the hadronic light-cone wave functions may be able to shed some light on several problems concerning the applicability of perturbative QCD in high momentum transfer region. The higher helicity states are likely the sources for the "'helicity non-conserving" behaviors [26] observed in $\mathrm{pp}^{\uparrow}$ scattering [27] and in $\pi \mathrm{N} \rightarrow \rho \mathrm{N}$ process [28]. The Melosh rotation also has implications in the spin content of hadrons. It has been shown in ref.[14] that the observed small value of the integrated spin structure function for protons, i.e., the spin EMC data [29], could be naturally understood within the naive quark model by taking into account the effect from Melosh rotation based on the facts that deep inelastic process probes the light-cone quarks other than the instant-form quarks $[1-2,21,30]$, and that the spin of the proton is the sum of the Melosh rotated light-cone spin of the individual quarks other than simply the sum of the light-cone spin of the quarks directly [31-32]. Thereby the effects from the Melosh rotation need more careful considerations. 
In summary, we showed, by using the pion as an example, that the spin structure of hadrons in light-cone formalism is quite different from that in the $\mathrm{SU}(6)$ naive quark model in considering the effect from the Melosh rotation. One example is the existence of the higher helicity states in the light-cone wave function for hadrons besides the ordinary helicity states. It is shown that some low energy properties of the pion, such as the electromagnetic form factor, the charged mean square radius, and the weak decay constant, could be interrelated by the harmonic oscillator wave function in the lightcone representation with very reasonable parameters by taking into account the contributions from the higher helicity states.

\section{Acknowledgement}

The author acknowledges many helpful discussions with Prof.T.Huang and Prof.Q.-X.Shen. He also thanks Prof.H.J.Weber for drawing his attention to the light-cone quark model and the Melosh rotation. The hospitality and support from Prof.W.Greiner at the Institut für Theoretische Physik der Universität Frankfurt are grateful. 


\section{References}

1. Brodsky, S.J.: In: Lectures on lepton nucleon scattering and quantum chromodynamics. Jaffe, A., Ruelle, D.(eds.), p.255, Boston: Birkhäuser 1982

Brodsky, S.J., Lepage, G.P.: In: Perturbative quantum chromodynamics. Mueller, A.H.(ed.), p.93, Singapore: World Scientific 1989 and references therein

2. Lepage, G.P. Brodsky, S.J.: Phys.Rev.D22, 2157(1980);

Brodsky, S.J., Huang,T., Lepage, G.P.: In: Particle and fields. Capri, A.Z., Kamal, A.N.(eds.), p.143, New York: Plenum 1983;

Lepage, G.P., Brodsky, S.J., Huang, T., Mackenzie, P.B.: ibid., p.83

3. Isgur, N., Llewellyn Smith, C.H.: Phys.Rev.Lett.52, 1080(1984)

4. Jacob, O.C., Kisslinger, L.S.: Phys.Rev.Lett.56, 225(1986)

5. Dziembowski, Z., Mankiewicz, L.: Phys.Rev.Lett.58, 2175(1987);

Dziembowski, Z.: In: Nuclear and particle physics on the light cone. Johnson, M.B., Kisslinger, L.S.(eds.), p.166, Singapore: World Scientific 1989

6. Huang, T.: Nucl.Phys.B(Proc.Suppl.)7, 320(1989);

Huang, T., Shen, Q.-X.: Z.Phys.C-Particles and Fields 50, 139(1991)

7. Isgur, N., Llewellyn Smith, C.H.: Nucl.Phys.B317, 526(1989);

Phys.Lett.B217, 535(1989);

see also, Isgur, N.: Nucl.Phys.A497, 229c(1989)

8. Dziembowski, Z.: Phys.Rev.D37, 778(1987). In this paper the term $b[\zeta-x(1-x)]$ in $(8)$ should be $2 \zeta^{2} x(1-x)$ 
9. Kisslinger, L.S., Jacob, O.C.: In: Nuclear and particle physics on the light cone. Johnson, M.B., Kisslinger, L.S.(eds.), p.322, Singapore: World Scientific 1989;

Jacob, O.C., Kisslinger, L.S.: Phys.Lett.B243, 323(1990)

10. Terent'ev, M.V.: Yad.Fiz.24, 207(1976) [Sov.J.Nucl.Phys.24, 106(1976)]

11. Karmanov, V.A.: Nucl.Phys.B166, 378(1980)

12. Chung, P.L., Coester, F., Polyzou, W.N.: Phys.Lett.B205, 545(1988)

13. Melosh, H.J.: Phys.Rev.D9, 1095(1974)

14. Ma, B.-Q.: J.Phys.G17, L53(1991)

15. Wigner, E.: Ann.Math.40, 149(1939)

16. See, Bjorken, J.D., Kogut, J.B., Soper, D.: Phys.Rev.D3, 1382(1971) and references therein

17. Kondratyuk, L.A., Terent'ev, M.V.: Yad.Fiz.31, 1087(1980) [Sov.J.Nucl.Phys.31, 561(1980)]

18. See, e.g., Elementary Particle Theory Group, Peking University: Acta Phys.Sin.25, 415(1976); Isgur, N.: In: The new aspects of subnuclear physics. Zichichi, A.(ed.), p.107, New York: Plenum 1980 and references therein

19. Dally, E.B., Hauptman, J.M., Kubic, J., Stork, D.H., Watson, A.B., Guzik, Z., Nigmanov, T.S., Riabtsov, V.D., Tsyganov, E.N., Vodopianov, A.S., Beretvas, A., Grigorian., A., Tompkins, J.C., Toohig, T.E., Wehmann, A.A., Poirier, J.A., Rey, C.A., Volk, J.T., Rapp, P.D., Shepard, P.F.: Phys.Rev.Lett.48, 375(1982)

20. Drell, S.D., Yan, T.-M.: Phys.Rev.Lett.24, 181(1970);

West, G.: Phys.Rev.Lett.24, 1206(1970) 
21. Ma, B.-Q.: Ph.D. Dissertation, Peking University 1989;

Ma, B.-Q., Sun, J.: J.Phys.G16, 823(1990); Int.J.Mod.Phys.A6, 345(1991);

Ma, B.-Q.: Phys.Rev.C43, 2821(1991)

22. Sawicki, M.: Phys.Rev.D46, 474(1992)

23. Amendila, S.R., Badelek, B., Batignani, G., Beck, G.A., Bedeschi, F., Bellamy, E.H., Bertolucci, E., Bettoni, D., Bilokon, H., Bologna, G., Bosisio, L., Bradaschia, C., Budinich, M., Codino, A., Counihan, M.J., Dell'orso, M., D'ettorre Piazzoli, B., Fabbri, F.L., Fidecaro, F., Foa, L., Focardi, E., Frank, S.G.F., Giazotto, A., Giorgi, M.A., Green, M.G., Harvey, J., Heath, G.P., Landon, M.P.J., Laurelli, P., Liello, F., Mannocchi, G., March, P.V., Marrocchesi, P.S., Menasce, D., Menzione, A., Meroni, E., Milotti, E., Moroni, L., Picchi, P., Ragusa, F., Ristori, L., Rolandi, L., Saltmarsh, C.G., Saoucha, A., Satta, L., Scribano, A., Spillantini, P., Stefanini, A., Storey, D., Strong, J.A., Tenchini, R., Tonelli, G., Von Schlippe, W., Van Herwijnen, E., Zallo, A.: Phys.Lett.B146, 116(1984); Nucl.Phys.B277, 168(1986)

24. Bedek, C.J., Brown, C.N., Holmes, S.D., Kline, R.V., Ripkin, F.M., Raither, S., Sisterson, L.K., Browman, A., Hanson, K.M., Larson, D., Silverman, A.: Phys.Rev.D17, 1693(1978) and references therein

25. Hwa, R.C.: Phys.Rev.D22, 759(1980);

Hwa, R.C., Lam, C.S.: Phys.Rev.D26, 2338(1982);

See, also, Zhu, W., Shen, J.G.: Phys.Rev.C41, 1674(1990)

26. Farrar, G.R.: Phys.Rev.Lett.56, 1643(1986)

27. Cameron, P.R., et al.: Phys.Rev.D32, 3070(1985)

28. Heppelmann, S., et al.: Phys.Rev.Lett.55, 1824(1985)

29. EMC, Ashman, J., Badelek, B., Baum, G., Beaufays, J., Bee, C.P., Benchouk, C., Bird, I.G., Brown, S.C., Caputo, M.C., Cheung, H.W.K., 
Chima, J., Ciborowski, J., Clifft, R.W., Coignet, G., Combley, F., Court, G., D'agostini, G., Drees, J., Düren, M., Dyce, N., Edwards, A.W., Edwards, M., Ernst, T., Ferrero, M.I., Francis, D., Gabathuler, E., Gajewski, J., Gamet, R., V.Gibson, V., Gillies, J., Grafström, P., Hamacher, K., Von Harrach, D., Hayman, P., Holt, J.R., Hughes, V.W., Jacholkowska, A., Jones, T., Kabuss, E.M., Korzen, B., Krüner, U., Kullander, S., Landgraf, U., Lanske, D., Lettenström, F., Lindqvist, T., Loken, J., Matthews, M., Mizuno, Y., Mönig, K., Montanet, F., Nassalski, J., Niinikoski, T., Norton, P.R., Oakham, G., Oppenheim, R.F., Osborne, A.M., Papavassiliou, V., Pavel, N., Peroni, C., Peschel, H., Piegaia, R., Pietrzyk, B., Pietrzyk., U., Povh, B., Renton, P., Rieubland, J.M., Rijllart, A., Rith, K., Rondio, E., Ropelewski, L., Salmon, D., Sandacz, A., Schröder, T., Schüler, K.P., Schultze, K., Shibata, T.-A., Sloan, T., Staiano, A., Stier, H., Stock, J., Taylor, G.N., Thompson, J.C., Walcher, T., Wheeler, S., Williams, W.S.C., Wimpenny, S.J., Windmolders, R., Womersley, W.J., Ziemons, K.: Phys.Lett.B206, 364(1988);

Nucl.Phys.B328, 1(1989)

30. Drell, S.D., Levy, D.J., Yan, T.-M.: Phys.Rev.187, 2159(1969);

Phys.Rev.D1, 1035(1970);

Drell, S.D., Yan, T.-M.: Ann.Phys.66, 578(1971)

31. Coester, F.: Helv.Phys.Acta 38, 7(1965); In: Constraint's theory and relativistic dynamics. Longhi, G., Lusanna, L.(eds.), p.159, Singapore: World Scientific 1987; In: The three-body force in the three-nucleon system. Berman, B.L., Gibson, B.F.(eds.), p.472, New York: Springer 1986

32. Chung, P.L., Coester, F., Keister, B.D., Polyzou, W.N.: Phys.Rev.C37, 2000(1988); see, also, Keister, B.D.: In: Nuclear and particle physics on the light cone. Johnson, M.B., Kisslinger, L.S.(eds.), p.439, Singapore: World Scientific 1989 


\section{Figure captions}

1. Fig.1. Pion form factors calculated with the pion wave functions in the three prescriptions at low $Q^{2}$. The solid, dashed, and dotted curves are the corresponding results from the BHL, TK, and CCP wave functions with the parameters $m$ and $\beta$ being: $m=330 \mathrm{MeV}$ and $\beta=290 \mathrm{MeV}$ for the BHL prescription; $m=330 \mathrm{MeV}$ and $\beta=280 \mathrm{MeV}$ for the TK prescription; and $m=330 \mathrm{MeV}$ and $\beta=270 \mathrm{MeV}$ for the CCP prescription. The data are taken from ref.[23].

2. Fig.2. Pion form factors calculated with the pion wave functions in the three prescriptions at high $Q^{2}$. The corresponding parameters for the curves are the same as those in fig.1. The curves labeled " $\lambda_{1}+\lambda_{2}=0 "$ are the contributions from the $\lambda_{1}+\lambda_{2}=0$ component wave functions in the corresponding prescription. If the $\lambda_{1}+\lambda_{2}= \pm 1$ components are ignored, the calculated results should be approximately twice the magnitude of the curves labeled "' $\lambda_{1}+\lambda_{2}=0$ " due to the normalization condition. The curves labeled "' $\mathcal{M}=1$ " are obtained by turning off the effect from the Melosh rotation. The unlabeled curves are the contributions from the full (i.e., the $\lambda_{1}+\lambda_{2}=0$ and $\lambda_{1}+\lambda_{2}= \pm 1$ components) wave functions. The data are taken from ref.[24]. 